\title{
Postconditioning attenuates myocardial ischemia-reperfusion injury by inhibiting complement activation and upregulation of miR-499
}

\author{
ZHENG HUANG $^{1 *}$, YAN HE $^{2,3 *}$, QING-JIE LI ${ }^{1}$, HONG WEN ${ }^{2,3}$, XIN-YUE ZHANG ${ }^{2}$, \\ RONG-HUI TU ${ }^{2,3}$ and GUO-QIANG ZHONG ${ }^{1,3}$
}

Departments of ${ }^{1}$ Cardiology and ${ }^{2}$ Geriatric Cardiology, First Affiliated Hospital, Guangxi Medical University;

${ }^{3}$ Guangxi Key Laboratory of Precision Medicine in Cardio-Cerebrovascular Diseases Control

and Prevention, Nanning, Guangxi 530021, P.R. China

Received April 8, 2020; Accepted February 22, 2021

DOI: $10.3892 /$ etm.2021.10116

\begin{abstract}
The complement system plays a vital role in myocardial ischemia/reperfusion (I/R) injury. microRNA (miR)-499 is involved in the cardioprotection of ischemic postconditioning (IPostC). The present study aimed to study the role of the complement system and miR-499 in IPostC. Rat hearts were subjected to coronary ligation for $30 \mathrm{~min}$, followed by reperfusion for $2 \mathrm{~h}$. IPostC was introduced at the onset of reperfusion with three cycles of reperfusion for $30 \mathrm{sec}$ and coronary artery occlusion for $30 \mathrm{sec}$. To study the role of miR-499 in IPostC, adeno-associated virus (AAV) vectors of miR-499-5p (AAV-miR-499-5p) and miR-499-5p-sponge (AAV-miR-499-5p-sponge) were transfected via tail vein injection, followed by IPostC protocols. Cardiac injury as well as the status of local and systemic complement activation and inflammation were assessed. IPostC significantly attenuated I/R-induced rat cardiomyocyte apoptosis and
\end{abstract}

Correspondence to: Dr Rong-Hui Tu, Department of Geriatric Cardiology, First Affiliated Hospital, Guangxi Medical University, 22 Shuangyong Road, Nanning, Guangxi 530021, P.R. China

E-mail: lily072trh@sina.com

Dr Guo-Qiang Zhong, Department of Cardiology, First Affiliated Hospital, Guangxi Medical University, 22 Shuangyong Road, Nanning, Guangxi 530021, P.R. China

E-mail: huangzheng07@sina.cn

*Contributed equally

Abbreviations: AAV, adeno-associated virus; C3a, complement component 3a; C5a, complement component 5a; I/R, ischemia/ reperfusion; IPostC, ischemic postconditioning; miRNA/miR, microRNA; MIRI, myocardial I/R injury; RT-qPCR, reverse transcription-quantitative polymerase chain reaction; TTC, triphenyl tetrazolium chloride

Key words: postconditioning, miR-499; myocardial ischemia/ reperfusion injury, the complement system, inflammation the myocardial infarct size. These beneficial effects were accompanied by decreased local and circulating complement component (C)3a and C5a levels, decreased inflammatory marker expression, decreased $\mathrm{NF}-\kappa \mathrm{B}$ signaling and increased cardiac miR-499 expression. AAV-miR-499-5p prevented local and systemic complement activation and inflammation as well as enhanced the cardioprotection of IPostC, whereas AAV-miR-499-5p-sponge produced the opposite effects. In summary, IPostC protected the rat myocardium against I/R injury, by inhibiting local and systemic complement activation; inflammation; NF-кB signaling; and upregulation of miR-499. As such, miR-499 may have a critical role in IPostC-mediated cardioprotection against I/R injury.

\section{Introduction}

Reperfusion following acute myocardial infarction is a double-edged sword. On one hand, reperfusion is the only option to salvage a dying myocardium (1). On the other hand, reperfusion can initiate myocardial ischemia/reperfusion (I/R) injury (MIRI), which produces additional serious tissue damage and may even become fatal to some patients receiving revascularization therapy (2).

The mechanisms underlying MIRI are poorly understood. Recently, complement activation has been found to play a pivotal role in MIRI (3-5). As part of the innate immune defense, the complement system consists of over 30 plasma and cell membrane proteins that are activated in a sequential manner, producing both local and systemic manifestations (6). For instance, excessive complement activation products complement component (C)3a and C5a are aggravating factors for myocardial necrosis and inflammatory cell infiltration (7). Conversely, inhibition of the complement cascade has been shown to significantly reduce MIRI $(8,9)$. Therefore, targeting the complement cascade may be a promising therapeutic strategy for treating I/R injury. Ischemic postconditioning (IPostC), defined as rapid, intermittent interruptions of blood flow in early reperfusion, has been shown to alleviate I/R injury in animal models and patients with acute myocardial infarction (10-12). However, the mechanisms of IPostC-related 
cardioprotection are not well understood. To the best of our knowledge, the role of critical novel pathways of I/R injury, such as activation of the complement system, have not been studied in myocardial IPostC.

MicroRNAs (miRNAs/miR) play a vital role in regulating ischemic injury (13). miR-499, a cardiac-enriched miRNA, participates in protection of the ischemic myocardium $(14,15)$. A recent study has reported that miR-499 has an antiapoptotic effect against MIRI (16). However, the potential role and mechanism of action underlying the cardioprotective effect of miR-499 in IPostC are poorly understood. The present study investigated the role of complement activation and the possible involvement of miR-499 in IPostC of the rat myocardium during $\mathrm{I} / \mathrm{R}$ injury.

\section{Materials and methods}

Animals. A total of 105 male adult Sprague-Dawley rats (8 weeks old, weighing 240-280 g) were collected from the Laboratory Animal Center of Guangxi Medical University. The rats were housed under standard laboratory conditions (temperature, $25 \pm 2^{\circ} \mathrm{C}$; relative humidity, $50 \pm 15 \% ; 12 \mathrm{~h}$ dark/light cycle) and rats were permitted ad libitum access to food and water. All animal protocols were performed in accordance with the Guide for the Care and Use of Laboratory Animals (National Institutes of Health, USA) and were approved by the Animal Care and Use Committee of Guangxi Medical University.

MIRI model. The rat MIRI model was established using a previously described method (17). Briefly, the rats were intraperitoneally injected with sodium pentobarbital $(2 \%, 50 \mathrm{mg} / \mathrm{kg})$ for general anesthesia and mechanically ventilated using a small animal respirator. A left parasternal incision was made in the fourth intercostal space to expose the heart. The left anterior descending coronary artery (LAD) was temporarily ligated at 2-3 $\mathrm{mm}$ below the lower edge of the left auricle using an 8-0 silk suture. A small plastic tube was inserted through the ligature to form a snare to enable reperfusion by reversing occlusion. Cardiac ischemia was induced by tightening the ligature around the plastic tube for $30 \mathrm{~min}$. Successful ligation was visually confirmed when the anterior wall of the left ventricle turned pale and by elevation of the ST segment on precordial leads of the electrocardiogram. Reperfusion was induced by loosening the ligation via the plastic tube, which lasted for $2 \mathrm{~h}$. IPostC was performed at the onset of reperfusion with three cycles of reperfusion for $30 \mathrm{sec}$, followed by coronary artery occlusion for $30 \mathrm{sec}$. Immediately after reperfusion, the rats were euthanized by cervical dislocation. Death of the rats was confirmed by the lack of a heart beat and respiration, and then the left ventricle of rats was harvested for further analysis.

Experimental grouping. The rats were randomly divided into following groups ( $n=15$ per group): i) Sham, in which the ligature was passed under the LAD, but not tied, and maintained for $150 \mathrm{~min}$; ii) $\mathrm{I} / \mathrm{R}$, in which the rats were subjected to $30 \mathrm{~min}$ of ischemia followed by $2 \mathrm{~h}$ of reperfusion; iii) IPostC, in which the rats underwent three cycles of $30 \mathrm{sec}$ of reperfusion and $30 \mathrm{sec}$ of ischemia, initiated immediately at the onset of reperfusion; iv) adeno-associated virus (AAV), in which the empty AAV vector $\left(1 \times 10^{12} \mathrm{v.g} . / \mathrm{rat}\right)$ was injected into the tail vein and did not receive any other treatment; v) AAV + IPostC, in which the empty AAV vector $\left(1 \times 10^{12} \mathrm{v.g} . / \mathrm{rat}\right)$ was injected into the tail vein, followed by the IPostC procedure after 4 weeks; vi) sponge + IPostC, which received the AAV vector of miR-499-5p-sponge (AAV-miR-499-5p-sponge, $1 \times 10^{12}$ v.g./rat) via tail vein injection, followed by the IPostC procedure after 4 weeks; vii) miR-499 + IPostC, which received the AAV vector of miR-499-5p (AAV-miR-499-5p, 1 x10 12 v.g./rat) followed by the IPostC procedure after 4 weeks. In total, 5 of the 105 rats used in this study were excluded: A total of 2 in the I/R group and 1 in the IPostC group died due to ventricular fibrillation; 1 in the AAV + IPostC group died due to cardiogenic shock during reperfusion; and 1 in the sponge + IPostC group died due to viral delivery failure. The results described are for the remaining 100 rats.

AAV transfection. The AAV-miR-499-5p (5'-GCTGTTAAG ACTTGCAGTGATGTTTAGCTCCTCTCCATGTGAACA TCACAGCAAGTCTGTGCTGC-3'), AAV-miR-499-5psponge (5'-AAACATCACTGCAAGTCTTAATATACAAAC ATCACTGCAAGTCTTAAACATCAAACATCACTGCAA GTCTTAATCTTCAAAACATCACTGCAAGTCTTAA-3'), and empty AAV vector (pHBAAV-U6-MCS-CMV-EGFP; Hanbio Biotechnology Co., Ltd.) as AAV control were constructed using previously described methods (18). The $\mathrm{AAV}$ vectors were injected through the tail vein at a dose of $1.0 \times 10^{12}$ genome copies per rat. Successful transfections were confirmed by detection of miR-499-5p expression in myocardial tissue at 4 weeks after injection.

Reverse transcription-quantitative PCR (RT-qPCR). Total RNA of ischemic myocardium was extracted using TRIzol ${ }^{\circledR}$ reagent (Invitrogen; Thermo Fisher Scientific, Inc.), and cDNA was synthesized from $1 \mu \mathrm{g}$ of total RNA using the RevertAid First Strand cDNA Synthesis kit (Thermo Scientific, Inc.) according to the manufacturer's instructions. qPCR was performed in 96-well plates using 2X SYBR Green qPCR ProMix (Guangzhou Yingzan Biological Technology Co., Ltd.) using an ABI 7300 Real-Time PCR System (Applied Biosystems). The thermocycling conditions were as follows: $95^{\circ} \mathrm{C}$ for $2 \mathrm{~min}, 40$ cycles of $95^{\circ} \mathrm{C}$ for $5 \mathrm{sec}$, and $60^{\circ} \mathrm{C}$ for $30 \mathrm{sec}$. All reactions were performed in triplicate. U6 was used as the reference gene. The relative expression of miRNA was determined using the $2^{-\Delta \Delta C q}$ method (19). The primer sequences used for RT-qPCR are listed in Table I.

Western blotting. Frozen rat myocardial tissue was lysed in radioimmunoprecipitation assay buffer (Beyotime Institute of Biotechnology) containing $1 \%$ phenylmethylsulfonyl fluoride (Beyotime Institute of Biotechnology) on ice. The protein concentration was measured using a bicinchoninic acid protein assay kit (Beyotime Institute of Biotechnology). Normalized protein samples $(30 \mu \mathrm{g})$ were resolved by $10 \%$ sodium dodecyl sulfate-polyacrylamide gel electrophoresis and transferred to PVDF (EMD Millipore). The membranes were blocked with $5 \%$ nonfat milk at room temperature for $1 \mathrm{~h}$, followed by incubation with primary antibodies against C3a (Abcam; 
Table I. The primer sequences for reverse transcriptionquantitative PCR.

\begin{tabular}{ll}
\hline Gene & \multicolumn{1}{c}{ Sequence } \\
\hline U6 forward & 5'-CTCGCTTCGGCAGCACA-3' \\
U6 reverse & 5'-AACGCTTCACGAATTTGCGT-3' \\
miR-499 forward & 5'-TTAAGACTTGCAGTGATGTTT-3' \\
miR-499 reverse & 5'-CAGTGCAGGGTCCGAGGTAT-3' \\
miRT Random $^{\text {Primer }}$ & 5'-GTCGTATCCAGTGCAGGGTCC \\
\hline
\end{tabular}

miR, microRNA. ${ }^{\mathrm{a}}$ Used in reverse transcription for cDNA synthesis.

cat. no. 171080; 1:1,000), C5a (Abcam; cat. no. 202039; 1:1,000), NF-кB p65 (Cell Signaling Technology, Inc.; cat. no. 8242; 1:1,000), TNF- $\alpha$ (Cell Signaling Technology, Inc.; cat. no. 11948; 1:1,000), IL-1 $\beta$ (Abcam; cat. no. 200478; 1:1,000), IL-6 (Abcam; cat. no. 208113; 1:1,000) and $\beta$-actin (Abcam; cat. no. 8226; 1:1,000) overnight at $4^{\circ} \mathrm{C}$. The membranes were washed three times with Tris-buffered saline containing $0.1 \%$ Tween-20 and then incubated with infrared dye-conjugated secondary antibodies (LI-COR Biosciences; cat. no. 926-32211; 1:10,000) for $1 \mathrm{~h}$ at room temperature. Protein bands were detected using an Odyssey Infrared Imaging System (LI-COR Biosciences) and the relative intensity of the bands was quantified using ImageJ v1.8.0 software (National Institutes of Health).

ELISAs. A total of $2 \mathrm{~h}$ after reperfusion of the myocardium, $2 \mathrm{ml}$ of venous blood was collected into EDTA-coated tubes. After centrifugation at $2,000 \mathrm{xg}$ and $4^{\circ} \mathrm{C}$ for $10 \mathrm{~min}$, the plasma samples were collected and stored at $-80^{\circ} \mathrm{C}$ for further analysis. The plasma levels of C3a (cat. no. 08510r), C5a (cat. no. 08513r),

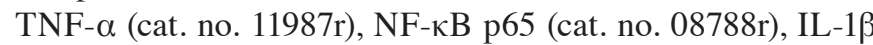
(cat. no.08055r) and IL-6 (cat. no. 04640r) were detected using commercial ELISA kits (Cusabio Technology LLC).

Triphenyl tetrazolium chloride (TTC) staining. The myocardial infarct area was assessed using TTC staining. Briefly, rat heart tissues were quickly isolated and washed with cold saline, then immediately frozen at temperature of $-70^{\circ} \mathrm{C}$ for $10 \mathrm{~min}$ and finally sliced into $2-\mathrm{mm}$ cross-sections from $2 \mathrm{~mm}$ below the ligation line. The slices were stained in $2 \%$ TTC solution (Beijing SolarbioScience and Technology Co., Ltd.) at $37^{\circ} \mathrm{C}$ for $20 \mathrm{~min}$ and then fixed in $4 \%$ paraformaldehyde at room temperature for $20 \mathrm{~min}$. The infarct size was quantified using Image J software.

TUNEL staining. TUNEL staining was performed on the myocardial sections to detect apoptotic cardiomyocytes, according to the manufacturer's instructions (Roche Diagnostics). Myocardial samples were fixed in $4 \%$ paraformaldehyde at $4^{\circ} \mathrm{C}$ for $24 \mathrm{~h}$ and embedded in paraffin. The dewaxed myocardial tissue sections were immersed in $3 \%$ hydrogen peroxide in methanol for $10 \mathrm{~min}$ at room temperature, and washed 3 times with PBS; then the tissue sections and proteinase $\mathrm{K}$ working solution incubated at $37^{\circ} \mathrm{C}$ for
$30 \mathrm{~min}$, which the tissue sections and TdT reaction mixture are incubated at $37^{\circ} \mathrm{C}$ for $2 \mathrm{~h}$. After washing 3 times with PBS, the nuclei were counterstained with DAPI solution $(5 \mu \mathrm{g} / \mathrm{ml})$ at room temperature for $5 \mathrm{~min}$. A total of $50 \mu \mathrm{l}$ anti-fade mounting medium was added to TUNEL-positive cells, which were observed in five randomly selected visual fields using a fluorescence microscope (magnification, x200). The index of apoptosis was expressed as follows: Index of apoptosis=(number of apoptotic cardiomyocytes/total number of cardiomyocytes) x $100 \%$.

Statistical analysis. The data were presented as the mean \pm SD and analyzed using SPSS 13.0 (SPSS Inc.) software. One-way ANOVAs were performed using post hoc Tukey's multiple comparison tests. A P-value $<0.05$ was considered statistically significant.

\section{Results}

IPostC reduces $I / R$-induced myocardial complement activation. Generation of C3a and C5a is commonly involved in all three complement activation pathways (20). Therefore, the present study examined the effects of IPostC on complement activation by detecting the expression of $\mathrm{C} 3 \mathrm{a}$ and $\mathrm{C} 5 \mathrm{a}$ in the rat myocardium. Western blot analysis showed that the expression levels of $\mathrm{C} 3 \mathrm{a}$ and $\mathrm{C} 5 \mathrm{a}$ were significantly increased in the rat myocardium following I/R injury, whereas IPostC attenuated the C3a and C5a upregulation following I/R (Fig. 1).

IPostC reduces the I/R-induced myocardial inflammatory response. Previous studies have demonstrated that activation of the complement system promotes inflammation in heart, kidney and brain tissues (21-23). To determine whether the inhibitory effect of IPostC on the complement system affects the inflammatory response in the myocardium the expression of the proinflammatory cytokines TNF- $\alpha$, IL- $1 \beta$ and IL- 6 was examined. I/R significantly increased the expression levels of TNF- $\alpha$, IL- $1 \beta$ and IL-6, as measured by western blot analyses compared to the sham group. IPostC then significantly reduced the expression levels of these inflammatory cytokines in the rat myocardium, compared with the I/R group, although this did not reach the expression levels observed in the sham group (Fig. 2).

IPostC reduces $I / R$-induced $N F-\kappa B$ activation. To explore the underlying mechanism of action behind the anti-inflammatory effect of IPostC, the present study examined the expression levels of $\mathrm{NF}-\kappa \mathrm{B}$ p 65 , a transcription factor critical for the activation of the complement system and proinflammatory cytokines. I/R induced a significant increase in the $N F-\kappa B$ p65 expression levels in the rat myocardium compared with the sham group, which was partially attenuated by IPostC (Fig. 3).

miR-499 reduces the expression of complement factors and inflammatory cytokines in the IPost $C$ rat myocardium. Previous studies have suggested that miR-499 has a protective effect against myocardial ischemia $(14,15,24)$. In order to further understand the role of miR-499 in myocardial IPostC, the present study transfected rats with empty AAV, 


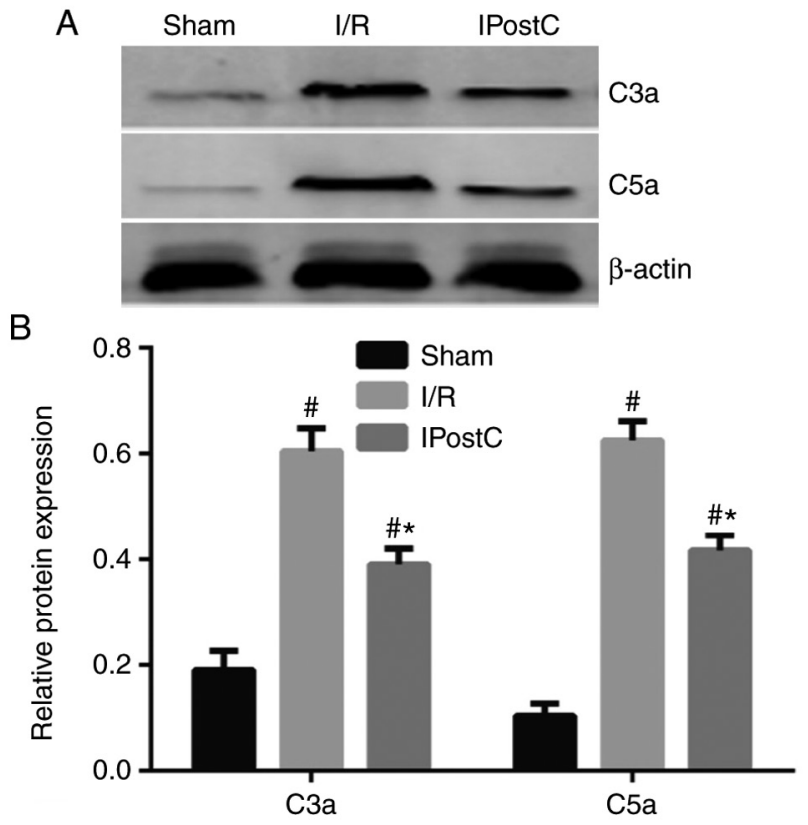

Figure 1. IPostC reduces I/R-induced expression of $\mathrm{C} 3 \mathrm{a}$ and $\mathrm{C} 5 \mathrm{a}$ in the rat myocardium. (A) Western blotting showing the expression of C3a and C5a under I/R injury and IPostC, and (B) quantification of C3a and C5a expression. Data are presented as the mean $\pm \mathrm{SD}, \mathrm{n}=4$. The experiments were repeated three times. ${ }^{\text {}} \mathrm{P}<0.05$ vs. Sham; ${ }^{\mathrm{P}} \mathrm{P}<0.05$ vs. I/R. C, complement component; I/R, ischemia/reperfusion; IPostC, ischemic postconditioning.

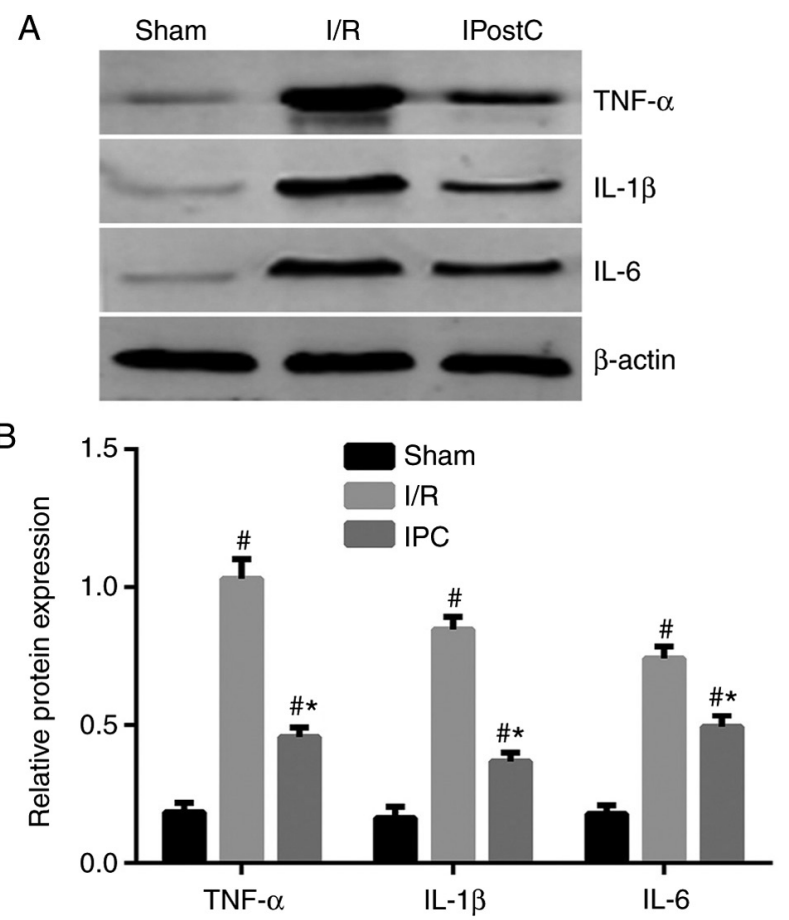

Figure 2. IPostC reduces the I/R-induced expression of TNF- $\alpha$, IL- $1 \beta$ and IL-6 in the rat myocardium. (A) Western blotting of rat myocardial tissue showing the expression of TNF- $\alpha$, IL-1 $\beta$ and IL-6 under Sham, I/R injury and IPostC conditions, and (B) quantification of TNF- $\alpha$, IL-1 $\beta$ and IL-6 expression. Data are presented as the mean $\pm \mathrm{SD}, \mathrm{n}=4$. The experiments were repeated three times. ${ }^{\#} \mathrm{P}<0.05$ vs. Sham; ${ }^{*} \mathrm{P}<0.05$ vs. I/R. I/R, ischemia/ reperfusion; IPostC, ischemic postconditioning.

AAV-miR-499-5p and AAV-miR-499-5p-sponge, and then subjected the rats to MIRI protocols with or without IPostC.
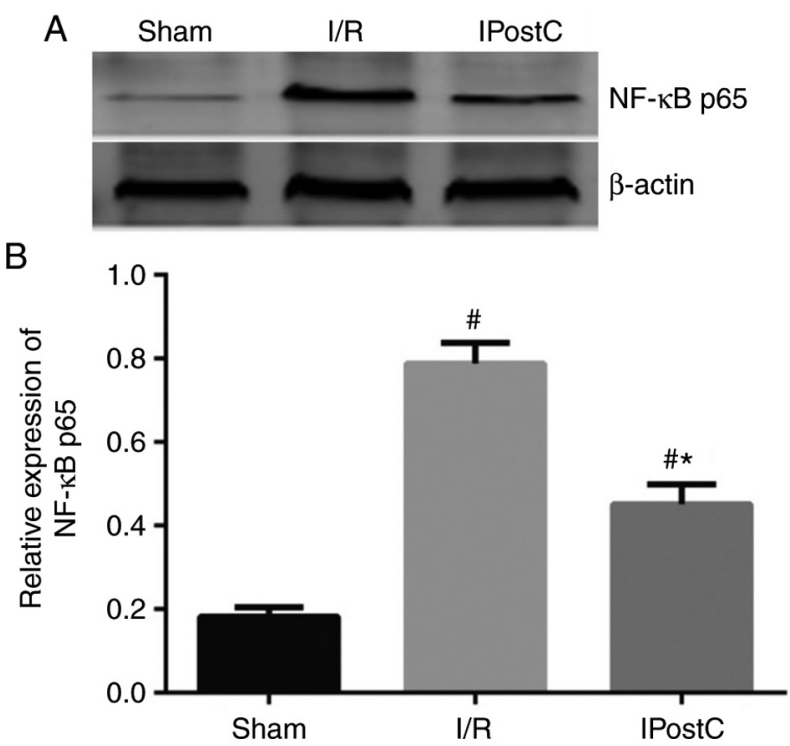

Figure 3. IPostC reduces $\mathrm{I} / \mathrm{R}$-induced $\mathrm{NF}-\kappa \mathrm{B}$ activation. (A) Western blotting showing the expression of NF-kB p65 under I/R injury and IPostC, and (B) quantification of $\mathrm{NF}-\kappa \mathrm{B}$ p65 expression. Data are presented as the mean $\pm \mathrm{SD}, \mathrm{n}=4$. The experiments were repeated three times. ${ }^{\#} \mathrm{P}<0.05$ vs. Sham; * $\mathrm{P}<0.05$ vs. I/R. I/R, ischemia/reperfusion; IPostC, ischemic postconditioning.

None of the rats showed behavioral abnormalities following injection of the AAV vectors. RT-qPCR confirmed the successful AAV transfection of the agents in the rat myocardium in all animals, treated either with or without IPostC (Fig. 4A). Compared with the sham group, the expression of miR-499 was significantly decreased in the I/R group, but it was significantly increased in the IPostC group (Fig. 4A). Notably, in rats receiving IPostC, AAV-miR-499-5p-sponge significantly increased the expression levels of C3a and C5a (Fig. 4B) as well as the proinflammatory cytokines TNF- $\alpha$, IL-1 $\beta$ and IL-6 (Fig. 4B), compared with the rats receiving empty AAV. In contrast, AAV-miR-499-5p significantly reversed the upregulation of complement factors and inflammatory cytokines in the IPostC rat myocardium (Fig. 4B). Taken together, these results strongly support a protective effect of miR-499 in the IPostC rat myocardium, in part, by inhibiting the upregulation of complement factors and inflammatory cytokines.

miR-499 inhibits $N F-\kappa B$ activation in the rat myocardium treated with IPostC. The present study further examined the effect of miR-499 on NF- $\kappa \mathrm{B}$ activation during IPostC. The expression of NF- $\kappa \mathrm{B}$ p 65 was not affected by the AAV (Fig. 5; AAV + IPostC). However, transfection with AAV-miR-499-5p-sponge significantly increased NF- $\mathrm{B}$ p65 expression (Fig. 5; sponge + IPostC vs. IPostC), whereas transduction with AAV-miR-499-5p significantly inhibited NF-кB p65 expression (Fig. 5; miR-499 + IPostC vs. IPostC). Together, these results further indicated a protective role of miR-499 in myocardial IPostC, in part by inhibiting $\mathrm{NF}-\kappa \mathrm{B}$ p65 pathway activation.

miR-499 reduces circulating inflammatory cytokines in rats treated with IPostC. To further assess the effects of miR-499 

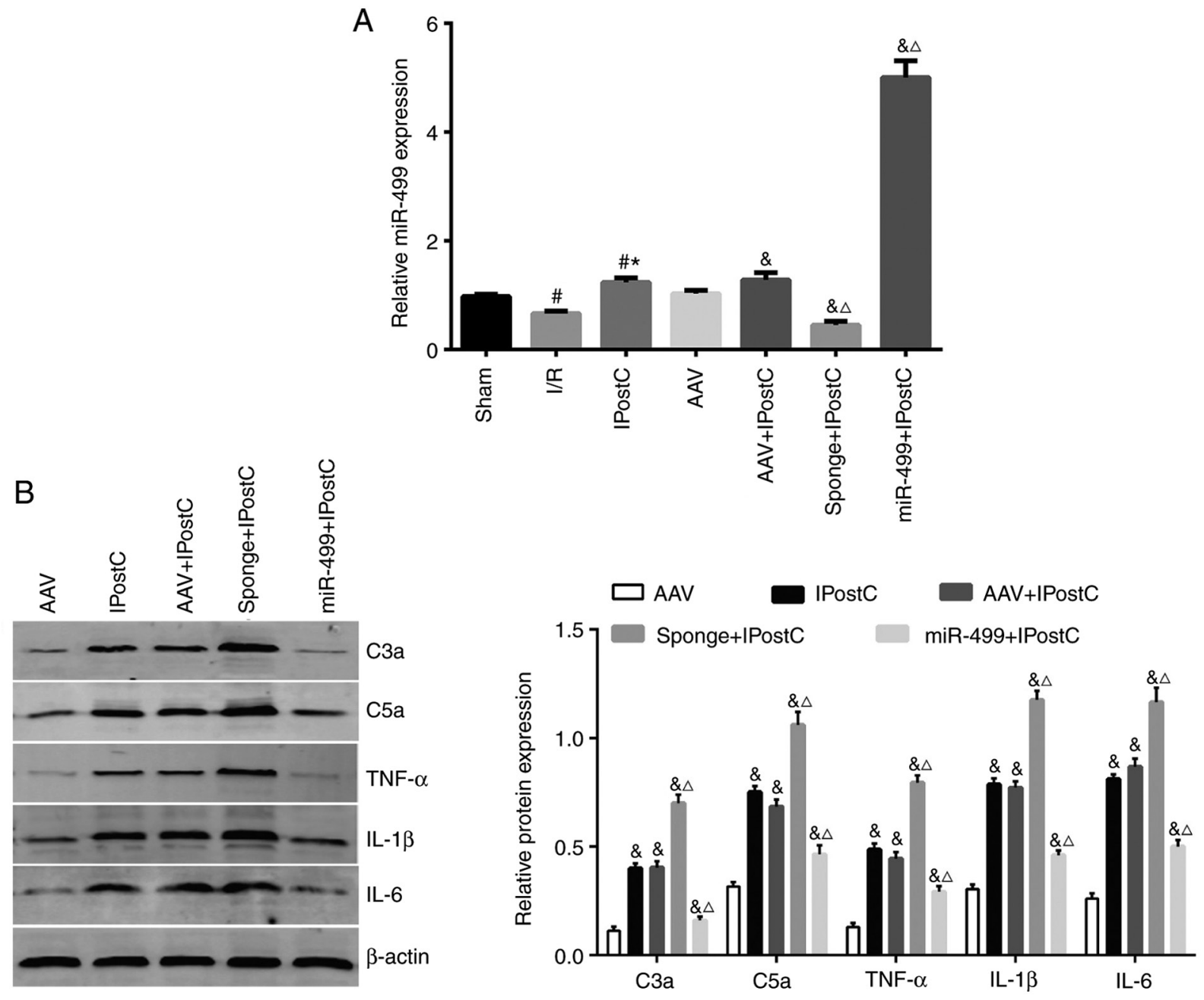

Figure 4. The effects of miR-499 on the expression of complement factors and inflammatory cytokines in the rat myocardium. (A) Reverse transcriptionquantitative PCR analysis of miR-499 expression levels in the rat myocardium receiving transduction of AAV-miR-499-5p, AAV-miR-499-5p-sponge and AAV control, with and without IPostC. The AAV injection was performed at 4 weeks prior to the PCR analysis. (B) Western blot analysis of C3a, C5a, TNF- $\alpha$, IL-1 $\beta$ and IL-6 expression in the treated rat myocardium. Quantification of the band intensity is presented beside the representative blots. Data are presented as the mean $\pm \mathrm{SD}, \mathrm{n}=4$. The experiments were repeated three times. ${ }^{*} \mathrm{P}<0.05$ vs. Sham; ${ }^{\mathrm{P}} \mathrm{P}<0.05$ vs. $/ \mathrm{R} ;{ }^{\circledR} \mathrm{P}<0.05$ vs. AAV; ${ }^{\wedge} \mathrm{P}<0.05$ vs. AAV $+\mathrm{IPostC}$. AAV, adeno-associated virus; $C$, complement component; $I / R$, ischemia/reperfusion; IPostC, ischemic postconditioning; miR, microRNA.

on the systemic inflammatory status during IPostC, the present study transfected rats with empty AAV, AAV-miR-499-5p and AAV-miR-499-5p-sponge and then measured the plasma levels of circulating complement factors and proinflammatory cytokines, including C3a, C5a, TNF- $\alpha$, IL-1 $\beta$, IL- 6 and NF- $\kappa B$ p65, using ELISAs. There were no significant differences in the plasma measurements between the IPostC and AAV + IPostC groups (Table II). In comparison with the IPostC and $\mathrm{AAV}+\mathrm{IPostC}$ groups, the plasma levels of C3a, C5a, TNF- $\alpha$, IL-1 $\beta$, IL- 6 and NF- $\kappa B$ p65 were all significantly higher in the rats receiving AAV-miR-499-5p-sponge and they were all significantly lower in the rats receiving AAV-miR-499-5p (Table II).

miR-499 reduces $I / R$-induced cardiomyocyte apoptosis during IPostC. The present study assessed cell apoptosis in the rat myocardium using TUNEL assays. Compared with the sham group, a significantly higher number of TUNEL-positive cells was detected in the I/R group (Fig. 6; brown nuclei in $\mathrm{I} / \mathrm{R}$ vs. Sham). IPostC treatment produced significantly fewer apoptotic cells than the I/R group (Fig. 6; IPostC vs. I/R). Transfection of the empty AAV had a minimal effect on cell apoptosis (Fig. 6; IPostC vs. AAV + IPostC). In contrast, AAV-miR-499-5p further reduced the number of apoptotic myocardiocytes in the IPostC rats (Fig. 6; miR-499 + IPostC vs. IPostC), whereas AAV-miR-499-5p-sponge significantly increased the number of TUNEL-positive cells in the IPostC myocardium (Fig. 6; sponge + IPostC vs. IPostC). Taken together, these results indicated a protective role of miR-499 against apoptosis in the IPostC rat myocardium.

miR-499 reduces the rat myocardial infarct size during IPostC. Finally, the present study assessed the role of miR-499 on IPostC by measuring the myocardial infarct size. There was virtually no visible myocardial infarction in the sham group (Fig. 7). In contrast, a large infarct zone was visible on the left ventricle of the I/R group (Fig. 7; I/R). Interestingly, IPostC significantly reduced the infarct myocardium area, with or without introduction of AAV compared with the I/R group (Fig. 7; IPostC vs. I/R; AAV + IPostC vs. I/R). 

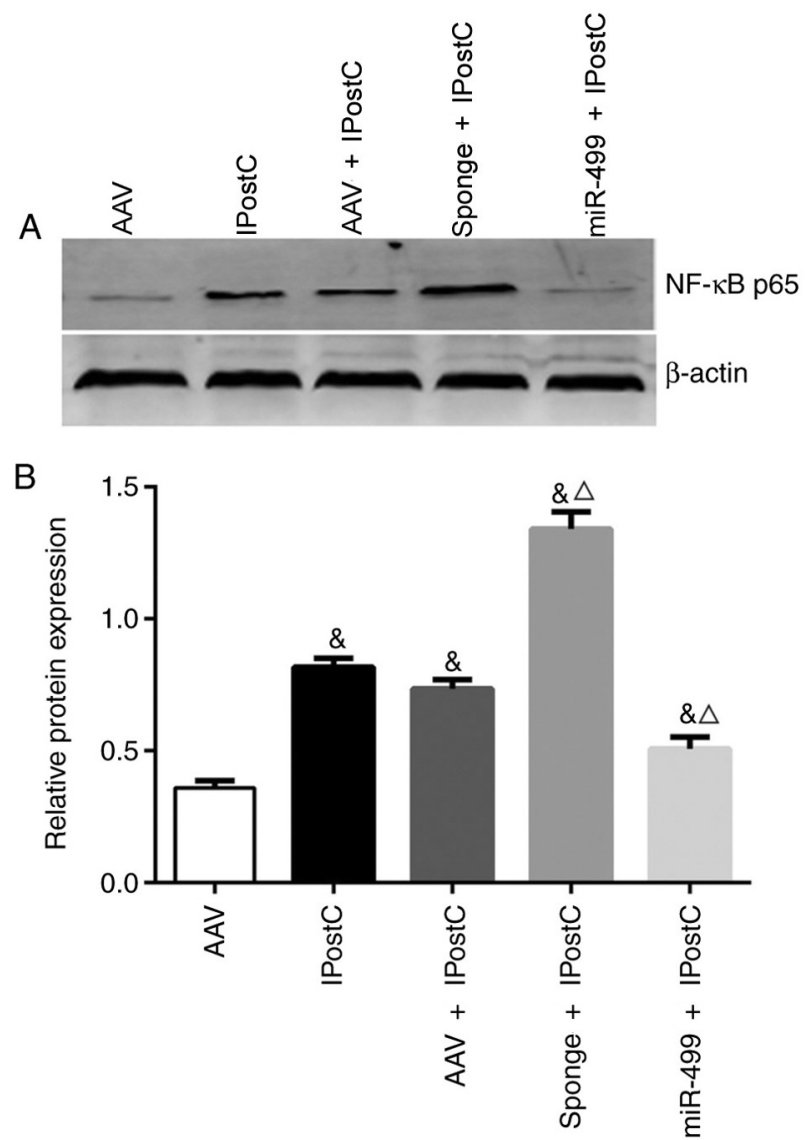

Figure 5. miR-499 inhibits NF- $\mathrm{B}$ activation in the rat myocardium treated with IPostC. (A) Western blotting of NF- $\mathrm{kB}$ p65 expression in the rat myocardium treated with IPostC and the various AAV vectors. (B) Quantification of $\mathrm{NF}-\kappa \mathrm{B}$ p65 expression in the rat myocardium in (A). Data are presented as the mean $\pm \mathrm{SD}, \mathrm{n}=4$. The experiments were repeated three times. ${ }^{\&} \mathrm{P}<0.05$ vs. AAV; ${ }^{\wedge} \mathrm{P}<0.05$ vs. AAV + IPostC. AAV, adeno-associated virus; IPostC ischemic postconditioning; miR, microRNA.

Notably, overexpression of the miR-499 further reduced the myocardial infarct zone following IPostC (Fig. 7; IPostC and $\mathrm{AAV}+$ IPostC vs. miR-499 + IPostC). Similarly, inhibition of miR-499 abolished the protective effect of IPostC on the myocardium and resulted in an increased infarct size (Fig. 7; IPostC and AAV + IPostC vs. sponge + IPostC).

\section{Discussion}

The major finding of the present study was that IPostC attenuated I/R-induced myocardial injury in a miR-499-dependent manner. IPostC effectively reduced I/R-induced complement activation, local and systemic inflammation, cardiomyocyte apoptosis and the myocardial infarct size. To the best of our knowledge, the present study is the first to demonstrate that miR-499 is an essential regulator of IPostC-mediated protection against I/R-induced myocardial injury, which is in part through local and systemic inhibition of complement activation, inflammation and NF- $\kappa \mathrm{B}$ signaling. Taken together, these results revealed a novel mechanism of action for miRNA-mediated IPostC protection.

Complement activation plays an important role in MIRI. Hill and Ward (25) first reported C3 deposition in an infarcted myocardium and revealed the relevance of complement activation in MIRI. In addition, Yasojima et al (26) have shown that endogenous C3 produced by the heart contributes to the degree of MIRI. Furthermore, it has been shown that suppressing specific components of the complement cascade protects against MIRI (27-30) and that ischemic preconditioning attenuates MIRI by inhibiting complement activation (31). However, to the best of our knowledge, the effect of IPostC on the complement system has not been reported. The present study found that the local and circulating levels of C3a and C5a were higher in the I/R group and lower in the IPostC group, cementing a critical role of complement factors in IPostC-induced cardioprotection.

Inflammatory responses represent a major pathological process leading to MIRI (32). An unchecked local inflammatory response has been observed in tissues subjected to I/R, whereas IPostC appears to inhibit the inflammatory response by reducing the expression or activation of local inflammatory mediators, or by decreasing inflammatory cytokine release $(33,34)$. Approaches that aim to inhibit these inflammatory responses following I/R could potentially lead to the attenuation of MIRI. Previous studies have shown that IPostC inhibits inflammation in renal tissues following I/R (35-37). The present study demonstrated a significant inflammatory response in rat cardiomyocytes following I/R injury and that IPostC significantly attenuated the inflammatory responses, partially due to the effect of miR-499 on reducing the expression of proinflammatory mediators in the IPostC rat myocardium.

The importance of complement activation is highlighted by its interaction with the inflammatory response and the $\mathrm{NF}-\kappa \mathrm{B}$ pathway, which together profoundly determine the outcome of I/R-induced tissue injury (38). Activated C3a and C5a attract proinflammatory leukocytes and promote the release of inflammatory cytokines during I/R (39). Once stimulated, such an inflammatory process tends to be self-propagative, resulting in sustained apoptosis and necrosis following MIRI (40). Recently, a correlation between complement activation and the $\mathrm{NF}-\kappa \mathrm{B}$ signaling pathway has been reported $(38,41)$. NF- $\kappa \mathrm{B}$ belongs to a family of transcription factors that plays a key role in regulating inflammatory responses and cell survival (42). Prior work has shown that IPostC inhibits I/R-induced inflammation and organ injury through $\mathrm{NF}-\kappa \mathrm{B}$ signaling $(35,43)$. However, the exact role of the complement-NF- $\kappa \mathrm{B}$ interaction in myocardial IPostC is unclear. The present results showed, for the first time, that IPostC simultaneously blocked complement activation and $\mathrm{NF}-\kappa \mathrm{B}$ signaling, supporting the notion that inhibition of both complement and $\mathrm{NF}-\kappa \mathrm{B}$ signaling may be involved in IPostC-induced cardioprotection.

miR-499 is most abundantly expressed in the heart and plays important roles in myocardial infarction and MIRI $(44,45)$. It has been shown that the miR-499 expression levels correlate with cardiomyocyte apoptosis and the severity of the infarction induced by I/R (15). For example, reperfusion reduces the expression levels of miR-499 in canine left ventricles and the miR-499 expression levels are negatively correlated with troponin $\mathrm{T}$ and creatine kinase-muscle/brain levels (24). However, the role of miR-499 in IPostC was unknown until a report in 2016 (16). In agreement with this previous report, the present study found that MIRI resulted in a reduction of miR-499, which was correlated with increased cardiomyocyte 
Table II. The plasma levels of complement factors and inflammatory cytokines in rats (mean \pm SD).

\begin{tabular}{lcccccc}
\hline Group & C3a $(\mu \mathrm{g} / \mathrm{ml})$ & C5a $(\mathrm{ng} / \mathrm{ml})$ & TNF- $\alpha(\mathrm{ng} / \mathrm{l})$ & IL-1 $\beta(\mathrm{ng} / \mathrm{ml})$ & IL-6 $(\mathrm{pg} / \mathrm{ml})$ & $\mathrm{NF}-\kappa B \mathrm{p} 65(\mathrm{pg} / \mathrm{ml})$ \\
\hline Sham & $887.5 \pm 67.6$ & $116.4 \pm 7.8$ & $276.4 \pm 5.7$ & $25.9 \pm 1.2$ & $70.3 \pm 4.1$ & $644.4 \pm 26.6$ \\
I/R & $1941.0 \pm 47.1^{\mathrm{a}}$ & $235.3 \pm 11.0^{\mathrm{a}}$ & $540.3 \pm 6.9^{\mathrm{a}}$ & $60.1 \pm 1.5^{\mathrm{a}}$ & $181.8 \pm 4.5^{\mathrm{a}}$ & $1282.0 \pm 26.6^{\mathrm{a}}$ \\
IPostC & $1333.9 \pm 69.8^{\mathrm{a}, \mathrm{b}}$ & $180.6 \pm 8.8^{\mathrm{a}, \mathrm{b}}$ & $355.7 \pm 13.2^{\mathrm{a}, \mathrm{b}}$ & $36.2 \pm 1.1^{\mathrm{a}, \mathrm{b}}$ & $130.1 \pm 7.7^{\mathrm{a}, \mathrm{b}}$ & $813.4 \pm 20.0^{\mathrm{a}, \mathrm{b}}$ \\
AAV + IPostC & $1366.7 \pm 51.2^{\mathrm{a}, \mathrm{b}}$ & $188.3 \pm 9.5^{\mathrm{a}, \mathrm{b}}$ & $360.0 \pm 14.4^{\mathrm{a}, \mathrm{b}}$ & $40.6 \pm 1.6^{\mathrm{a}, \mathrm{b}}$ & $131.0 \pm 8.1^{\mathrm{a}, \mathrm{b}}$ & $849.6 \pm 19.2^{\mathrm{a}, \mathrm{b}}$ \\
Sponge + IPostC & $1735.0 \pm 42.6^{\mathrm{c}, \mathrm{d}}$ & $212.3 \pm 9.4^{\mathrm{c}, \mathrm{d}}$ & $445.1 \pm 7.4^{\mathrm{c}, \mathrm{d}}$ & $46.9 \pm 1.3^{\mathrm{c}, \mathrm{d}}$ & $161.8 \pm 3.5^{\mathrm{c}, \mathrm{d}}$ & $975.5 \pm 15.8^{\mathrm{c}, \mathrm{d}}$ \\
miR-499 + IPostC & $1085.6 \pm 64.4^{\mathrm{c}, \mathrm{d}}$ & $154.8 \pm 9.7^{\mathrm{c}, \mathrm{d}}$ & $308.3 \pm 5.9^{\mathrm{c}, \mathrm{d}}$ & $31.5 \pm 0.7^{\mathrm{c}, \mathrm{d}}$ & $89.3 \pm 3.3^{\mathrm{c}, \mathrm{d}}$ & $742.4 \pm 17.8^{\mathrm{c}, \mathrm{d}}$ \\
\hline
\end{tabular}

$\mathrm{n}=4$. The experiments were repeated four times. ${ }^{\mathrm{a}} \mathrm{P}<0.05$ vs. Sham; ${ }^{\mathrm{b}}<0.05 \mathrm{vs} . \mathrm{I} / \mathrm{R} ;{ }^{\mathrm{c}} \mathrm{P}<0.05$ vs. IPostC; ${ }^{\mathrm{d}} \mathrm{P}<0.05$ vs. AAV $+\mathrm{IPostC} . \mathrm{AAV}$, adeno-associated virus; I/R, ischemia/reperfusion; IPostC, ischemic postconditioning; miR, micro.
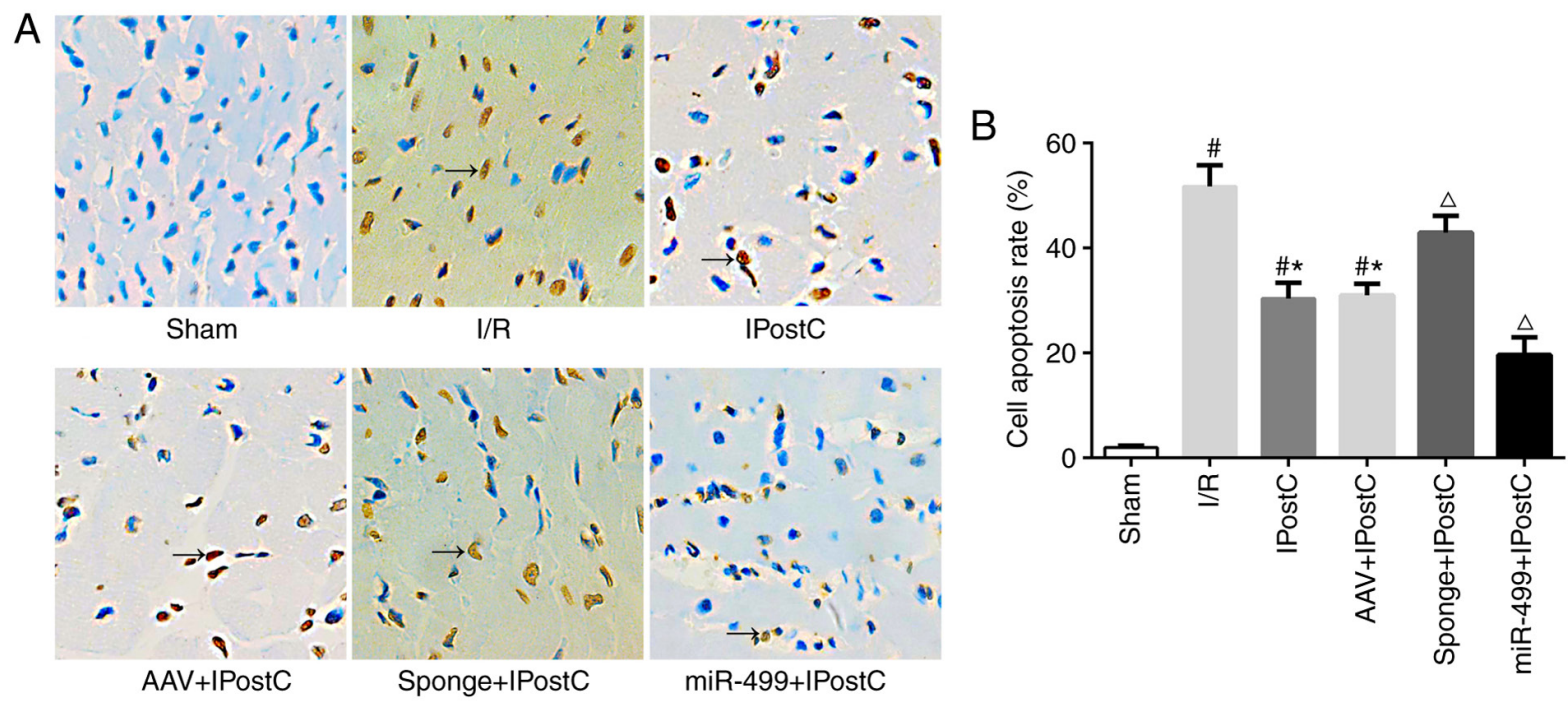

Figure 6. miR-499 protects against cardiomyocyte apoptosis during IPostC. (A) Representative micrographs of TUNEL staining in the rat myocardium (magnification, x200). Arrows indicate TUNEL-positive cells. (B) Quantitative analysis of the percentage of TUNEL-positive cardiomyocytes. Data are presented as the mean $\pm \mathrm{SD}, \mathrm{n}=4$. The experiments were repeated three times. ${ }^{\#} \mathrm{P}<0.05$ vs. Sham; ${ }^{*} \mathrm{P}<0.05$ vs. I/R; ${ }^{\circ} \mathrm{P}<0.05$ vs. AAV + IPostC. AAV, adeno-associated virus; $\mathrm{I} / \mathrm{R}$, ischemia/reperfusion; IPostC, ischemic postconditioning; miR, microRNA.

A
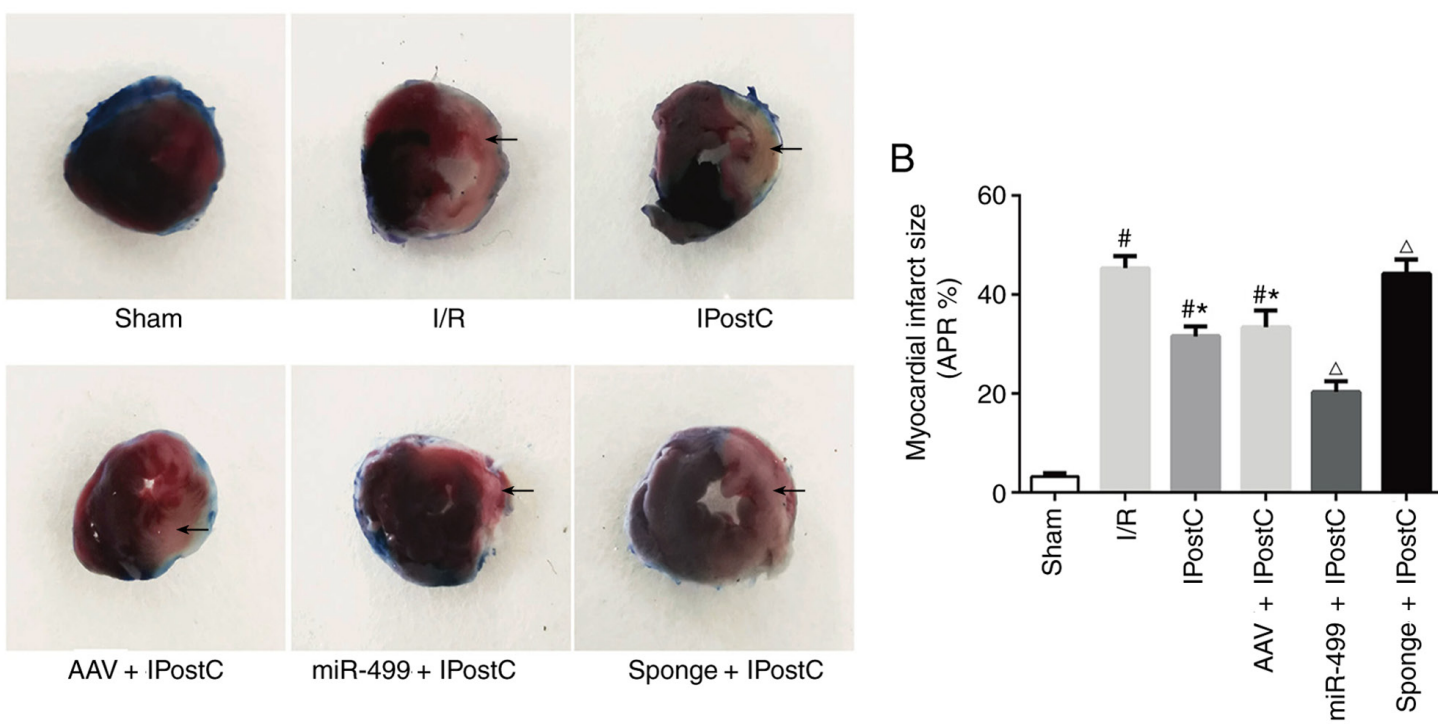

Figure 7. The myocardial infarct size in each group as detected using TTC staining. (A) Representative photographs of TTC staining of myocardial slices. The slices were taken from $2 \mathrm{~mm}$ below the ligation line (magnification, x200). (B) Quantitative analysis of the myocardial infarct size. Data are presented as the mean $\pm \mathrm{SD}, \mathrm{n}=4$. The experiments were repeated three times. ${ }^{*} \mathrm{P}<0.05$ vs. Sham; ${ }^{*} \mathrm{P}<0.05$ vs. I/R; ${ }^{\Delta} \mathrm{P}<0.05$ vs. AAV $+\mathrm{IPostC}$. AAV, adeno-associated virus; $\mathrm{I} / \mathrm{R}$, ischemia/reperfusion; IPostC, ischemic postconditioning; miR, microRNA; TTC, triphenyl tetrazolium chloride. 
apoptosis and an increased myocardial infarct size. Moreover, the converse changes were found in the IPostC myocardium. The present mechanistic studies revealed that IPostC led to an increased miR-499 level and that by manipulating the miR-499 level, critical pathways such as complement activation, inflammation and $\mathrm{NF}-\kappa \mathrm{B}$ signaling were affected. This resulted in significantly altered outcomes of myocardial protection following IPostC. Therefore, miR-499 plays a central role in IPostC-induced cardioprotection.

A primary limitation of the present study is that the effects of IPostC and miR-499 on cardiac function in ischemia-reperfusion rats were not evaluated. Previous studies have shown that IPostC improves the longitudinal contractile function of the reperfused myocardium in patients with acute myocardial infarction (46) and that miR-499 improves left ventricular function in a rat model of IPostC (16). Considering that cardiomyocyte apoptosis and myocardial necrosis are closely related to cardiac function, it is speculated that miR-499 may improve cardiac function in ischemia-reperfusion rats. Future functional studies using ultrasound or electrocardiography to evaluate the effect of miR-499 on live rats during IPostC are recommended.

In conclusion, miR-499 regulated IPostC-mediated protection against I/R-induced myocardial injury, in part by inhibiting the activation of local and systemic C3a and C5a; inflammation; and $\mathrm{NF}-\kappa \mathrm{B}$ signaling. The present data provided mechanistic evidence which could support the development of novel therapeutics aimed at harnessing IPostC-mediated cardioprotection against MIRI.

\section{Acknowledgements}

Not applicable.

\section{Funding}

This study was supported by grants from the National Natural Science Foundation of China (grant no. 81560068) and the Natural Science Foundation of Guangxi Province (grant no. 2015GXNSFAA139198).

\section{Availability of data and materials}

The datasets generated and analyzed during the present study are available from the corresponding author on reasonable request.

\section{Authors' contributions}

$\mathrm{ZH}$ and $\mathrm{YH}$ designed and performed experiments, analyzed data and co-wrote the paper. QJL, HW and XYZ performed experiments. RHT and GQZ designed experiments; provided research funding; and edited and revised the manuscript. All authors have read and provided final approval of the manuscript. ZH and RHT confirm the authenticity of all the raw data. All authors read and approved the final manuscript.

\section{Ethics approval and consent to participate}

All animal protocols were performed in accordance with the Guide for the Care and Use of Laboratory Animals (National
Institutes of Health, USA) and were approved by the Animal Care and Use Committee of Guangxi Medical University.

\section{Patient consent for publication}

Not applicable.

\section{Competing interests}

The authors declare that they have no competing interests.

\section{References}

1. Ibanez B, James S, Agewall S, Antunes MJ, Bucciarelli-Ducci C, Bueno H, Caforio ALP, Crea F, Goudevenos JA, Halvorsen S, et al: 2017 ESC guidelines for the management of acute myocardial infarction in patients presenting with ST-segment elevation: The Task Force for the management of acute myocardial infarction in patients presenting with ST-segment elevation of the European Society of Cardiology (ESC). Eur Heart J 39: 119-177, 2018.

2. Neri M, Riezzo I, Pascale N, Pomara C and Turillazzi E: Ischemia/reperfusion injury following acute myocardial infarction: A critical issue for clinicians and forensic pathologists. Mediators Inflamm 2017: 7018393, 2017.

3. Tang K, Cheng Y, Wu S, Liu L and Cheng L: Protective effect of C5 shRNA on myocardial ischemia-reperfusion injury in rats. Can J Physiol Pharmacol 90: 1394-1402, 2012.

4. Frangogiannis NG: The inflammatory response in myocardial injury, repair, and remodelling. Nat Rev Cardiol 11: 255-265, 2014.

5. Chun N, Haddadin AS, Liu J, Hou Y, Wong KA, Lee D, Rushbrook JI, Gulaya K, Hines R, Hollis T, et al: Activation of complement factor $\mathrm{B}$ contributes to murine and human myocardial ischemia/reperfusion injury. PLoS One 12: e0179450, 2017.

6. Bardhan M and Kaushik R: Physiology, complement cascade. In: StatPearls, Treasure Island, FL, 2021.

7. Busche MN and Stahl GL: Role of the complement components $\mathrm{C} 5$ and $\mathrm{C} 3 \mathrm{a}$ in a mouse model of myocardial ischemia and reperfusion injury. Ger Med Sci 8: Doc20, 2010.

8. Fu J, Lin G, Wu Z, Ceng B, Wu Y, Liang G, Qin G, Li J, Chiu I and Liu D: Anti-apoptotic role for C1 inhibitor in ischemia/reperfusion-induced myocardial cell injury. Biochem Biophys Res Commun 349: 504-512, 2006.

9. Pavlov VI, Tan YS, McClure EE, La Bonte LR, Zou C, Gorsuch WB and Stahl GL: Human mannose-binding lectin inhibitor prevents myocardial injury and arterial thrombogenesis in a novel animal model. Am J Pathol 185: 347-355, 2015.

10. Hao M, Zhu S, Hu L, Zhu H, Wu X and Li Q: Myocardial ischemic postconditioning promotes autophagy against ischemia reperfusion injury via the activation of the nNOS/AMPK/mTOR Pathway. Int J Mol Sci 18: 614, 2017.

11. Khan AR, Binabdulhak AA, Alastal Y, Khan S, Faricy-Beredo BM, Luni FK, Lee WM, Khuder S and Tinkel J: Cardioprotective role of ischemic postconditioning in acute myocardial infarction: A systematic review and meta-analysis. Am Heart J 168: 512-521.e4, 2014.

12. Aslan G, Gul HF, Tektemur A and Sahna E: Ischemic postconditioning reduced myocardial ischemia-reperfusion injury: The roles of melatonin and uncoupling protein 3 . Anatol $\mathrm{J}$ Cardiol 23: 19-27, 2020.

13. Ambros V: The functions of animal microRNAs. Nature 431: 350-355, 2004.

14. Li Y, Lu J, Bao X, Wang X, Wu J, Li X and Hong W: MiR-499-5p protects cardiomyocytes against ischaemic injury via anti-apoptosis by targeting PDCD4. Oncotarget 7: 35607-35617, 2016.

15. Wang JX, Jiao JQ, Li Q, Long B, Wang K, Liu JP, Li YR and Li PF: miR-499 regulates mitochondrial dynamics by targeting calcineurin and dynamin-related protein-1. Nat Med 17: 71-78, 2011.

16. Zhu J, Yao K, Wang Q, Guo J, Shi H, Ma L, Liu H, Gao W, Zou Y and $\mathrm{Ge} \mathrm{J}$ : Ischemic postconditioning-regulated miR-499 protects the rat heart against ischemia/reperfusion injury by inhibiting apoptosis through PDCD4. Cell Physiol Biochem 39: 2364-2380, 2016.

17. Zhong GQ, Tu RH, Zeng ZY, Li QJ, He Y, Li S, He Y and Xiao F: Novel functional role of heat shock protein 90 in protein kinase C-mediated ischemic postconditioning. J Surg Res 189: 198-206, 2014. 
18. Fan JJ, Gao B, Song AQ, Zhu YJ, Zhou J, Li WZ, Yin YY and Wu WN: Spinal cord NLRP1 inflammasome contributes to dry skin induced chronic itch in mice. J Neuroinflammation 17: 122, 2020.

19. Livak KJ and Schmittgen TD: Analysis of relative gene expression data using real-time quantitative PCR and the 2(-Delta Delta C(T)) method. Methods 25: 402-408, 2001

20. Haddad A and Wilson AM: Biochemistry, complement. In: StatPearls, Treasure Island, FL, 2021.

21. Shahini N, Michelsen AE, Nilsson PH, Ekholt K, Gullestad L, Broch K, Dahl CP, Aukrust P, Ueland T, Mollnes TE, et al: The alternative complement pathway is dysregulated in patients with chronic heart failure. Sci Rep 7: 42532, 2017.

22. Mocco J, Mack WJ, Ducruet AF, Sosunov SA, Sughrue ME, Hassid BG, Nair MN, Laufer I, Komotar RJ, Claire M, et al: Complement component $\mathrm{C} 3$ mediates inflammatory injury following focal cerebral ischemia. Circ Res 99: 209-217, 2006.

23. Danobeitia JS, Ziemelis M, Ma X, Zitur LJ, Zens T, Chlebeck PJ, Van Amersfoort ES and Fernandez LA: Complement inhibition attenuates acute kidney injury after ischemia-reperfusion and limits progression to renal fibrosis in mice. PLoS One 12 e0183701, 2017.

24. Qin H, Chen GX, Liang MY, Rong J, Yao JP, Liu H and Wu ZK: The altered expression profile of microRNAs in cardiopulmonary bypass canine models and the effects of mir-499 on myocardial ischemic reperfusion injury. J Transl Med 11: 154, 2013.

25. Hill JH and Ward PA: The phlogistic role of C3 leukotactic fragments in myocardial infarcts of rats. J Exp Med 133: 885-900, 1971.

26. Yasojima K, Kilgore KS, Washington RA, Lucchesi BR and McGeer PL: Complement gene expression by rabbit heart: Upregulation by ischemia and reperfusion. Circ Res 82: 1224-1230, 1998.

27. Kassimatis T, Qasem A, Douiri A, Ryan EG, Rebollo-Mesa I, Nichols LL, Greenlaw R, Olsburgh J, Smith RA, Sacks SH and Drage M: A double-blind randomised controlled investigation into the efficacy of Mirococept (APT070) for preventing ischaemia reperfusion injury in the kidney allograft (EMPIRIKAL): Study protocol for a randomised controlled trial. Trials 18: 255, 2017.

28. Vo AA, Zeevi A, Choi J, Cisneros K, Toyoda M, Kahwaji J, Peng A, Villicana R, Puliyanda D, Reinsmoen N, et al: A phase I/II placebo-controlled trial of C1-inhibitor for prevention of antibody-mediated rejection in HLA sensitized patients. Transplantation 99: 299-308, 2015.

29. Thielmann M, Marggraf G, Neuhäuser M, Forkel J, Herold U, Kamler M, Massoudy $\mathrm{P}$ and Jakob $\mathrm{H}$ : Administration of $\mathrm{C} 1$-esterase inhibitor during emergency coronary artery bypass surgery in acute ST-elevation myocardial infarction. Eur J Cardiothorac Surg 30: 285-293, 2006.

30. Smith PK, Carrier M, Chen JC, Haverich A, Levy JH, Menasché P, Shernan SK, Van de Werf F, Adams PX, Todaro TG and Verrier E: Effect of pexelizumab in coronary artery bypass graft surgery with extended aortic cross-clamp time. Ann Thorac Surg 82: 781-789, 2006.

31. Tanhehco EJ, Yasojima K, McGeer PL, McGeer EG and Lucchesi BR: Preconditioning reduces myocardial complement gene expression in vivo. Am J Physiol Heart Circ Physiol 279: H1157-H1165, 2000

32. Blancke F, Claeys MJ, Jorens P, Vermeiren G, Bosmans J, Wuyts FL and Vrints CJ: Systemic inflammation and reperfusion injury in patients with acute myocardial infarction. Mediators Inflamm 2005: 385-389, 2005.
33. Kong Y, Rogers MR and Qin X: Effective neuroprotection by ischemic postconditioning is associated with a decreased expression of RGMa and inflammation mediators in ischemic rats. Neurochem Res 38: 815-825, 2013.

34. Wu H, Lei S, Yuan J, Liu X, Zhang D, Gu X, Zhang L and Xia Z: Ischemic postconditioning downregulates Egr-1 expression and attenuates postischemic pulmonary inflammatory cytokine release and tissue injury in rats. J Surg Res 181: 204-212, 2013.

35. Chen H, Wang L, Xing BZ, Liu XH, Chen ZY, Weng XD, Qiu T and Liu L: Ischemic postconditioning attenuates inflammation in rats following renal ischemia and reperfusion injury. Exp Ther Med 10: 513-518, 2015.

36. Chen R, Zeng Z, Zhang YY, Cao C, Liu HM, Li W, Wu Y, Xia ZY, $\mathrm{Ma} \mathrm{D}$ and Meng QT: Ischemic postconditioning attenuates acute kidney injury following intestinal ischemia-reperfusion through Nrf2-regulated autophagy, anti-oxidation, and anti-inflammation in mice. FASEB J 34: 8887-8901, 2020.

37. Guo Q, Du X, Zhao Y, Zhang D, Yue L and Wang Z: Ischemic postconditioning prevents renal ischemia reperfusion injury through the induction of heat shock proteins in rats. Mol Med Rep 10: 2875-2881, 2014.

38. Lin Z, Lin H, Li W, Huang Y and Dai H: Complement component C3 promotes cerebral ischemia/reperfusion injury mediated by TLR2/NFKB activation in diabetic mice. Neurochem Res 43: 1599-1607, 2018

39. Peng Q, Li K, Smyth LA, Xing G, Wang N, Meader L, Lu B, Sacks SH and Zhou W: C3a and C5a promote renal ischemia-reperfusion injury. J Am Soc Nephrol 23: 1474-1485, 2012.

40. Gorsuch WB, Chrysanthou E, Schwaeble WJ and Stahl GL: The complement system in ischemia-reperfusion injuries. Immunobiology 217: 1026-1033, 2012.

41. Liu M, Wang H, Zhang J, Yang X, Li B, Wu C and Zhu Q: $\mathrm{NF}-\kappa \mathrm{B}$ signaling pathway-enhanced complement activation mediates renal injury in trichloroethylene-sensitized mice. J Immunotoxicol 15: 63-72, 2018.

42. Liu T, Zhang L, Joo D and Sun SC: NF- $\kappa$ B signaling in inflammation. Signal Transduct Target Ther 2: 17023, 2017.

43. Kin H, Wang NP, Mykytenko J, Reeves J, Deneve J, Jiang R, Zatta AJ, Guyton RA, Vinten-Johansen J and Zhao ZQ: Inhibition of myocardial apoptosis by postconditioning is associated with attenuation of oxidative stress-mediated nuclear factor-kappa B translocation and TNF alpha release. Shock 29: 761-768, 2008.

44. Adachi T, Nakanishi M, Otsuka Y, Nishimura K, Hirokawa G, Goto Y, Nonogi H and Iwai N: Plasma microRNA 499 as a biomarker of acute myocardial infarction. Clin Chem 56: 1183-1185, 2010

45. Sluijter JP, van Mil A, van Vliet P, Metz CH,Liu J, Doevendans PA and Goumans MJ: MicroRNA-1 and -499 regulate differentiation and proliferation in human-derived cardiomyocyte progenitor cells. Arterioscler Thromb Vasc Biol 30: 859-868, 2010.

46. Yang Z, Zhou Q, Fang Z, Yu L, Zhou J and Zhao B: Ischemic postconditioning improves longitudinal contractile function of the reperfused myocardium in patients with anterior wall acute myocardial infarction. Zhong Nan Da Xue Xue Bao Yi Xue Ban 44: 1397-1405, 2019 (In Chinese).

This work is licensed under a Creative Commons Attribution-NonCommercial-NoDerivatives 4.0 International (CC BY-NC-ND 4.0) License. 